\title{
Discontinuous feedback laws for infinite dimensional systems
}

\section{Laura Levaggi*}

Dipartimento di Matematica, Università di Genova, Via Dodecaneso, 35, 16146 Genova, Italy

E-mail: levaggiedima.unige.it

The application of discontinuous feedback laws to infinite-dimensional control systems, with particular reference to sliding motions, is discussed for both linear systems with distributed control and parabolic differential equations with Neumann boundary control. In the first case it is shown how, using differential inclusions and viability theory, it is possible to interpret the constrained motion on the sliding surface as a generalised solution of the discontinuous control problem. For the second class of systems a variational approach is considered. Under some growth assumptions it is shown that Faedo-Galerkin approximations of the evolution, satisfying the sliding constraint in the limit, do converge to an ideal sliding state.

Control Systems: Theory, Numerics and Applications 30 March - 1 April 2005

Rome

\footnotetext{
${ }^{*}$ Speaker.
} 


\section{Introduction}

Variable structure control methods and in particular sliding mode controls, are by now recognised as classical tools for the regulation of systems governed by ordinary differential equations in a finite dimensional setting. For an overview of the finite-dimensional theory see [17]. While being easy to design, they possess attractive properties of robustness and insensitivity with respect to disturbances and unmodeled dynamics. These characteristics are all the more important when dealing with infinite-dimensional systems. Recent research has been devoted to the extension of sliding mode control and therefore the use of discontinuous feedback laws, to the infinite-dimensional setting. The early works [10, 11, 13] were confined to some special classes of systems, but at present both theory and application of sliding mode control have been extended to a rather general setting [14, 12, 15]. In particular in [14] the key concept of equivalent control is extended to evolution equations governed by unbounded linear operators that generate $C_{0}$-semigroups.

As sliding motions are obtained by applying feedback laws which are discontinuous on the sliding surface, the question of how to define what is the meaning of solution for the closed loop is a crucial point. A generalised solution concept has been proposed in [5, 5] and a relationship between the equivalent control method and generalised solutions of infinite-dimensional systems with discontinuous right-hand side has been established, under some regularity assumptions. In Section 2 these results are extended to a more general setting by requiring less stringent hypotheses on the interaction between the evolution operator and the sliding surface. This allows for more flexibility in the construction of the sliding manifold and this is of primary importance for application purposes.

All the results in the above cited literature only take into consideration distributed control systems, i.e. they deal with bounded input operators. In Section 3 a variational approach in the direction of [19] is proposed in order to show the existence of sliding modes for a class of parabolic evolution equations with Neumann boundary control. The main assumptions made on the operator governing the evolution, are weak continuity and coerciveness, so that both linear and non-linear operators may fall into this setting. Then a Faedo-Galerkin method is used to construct a sequence of finite-dimensional approximations of the given problem. The main result then states that if for each approximation a control law is chosen to constrain the evolution in a boundary layer of a given sliding manifold, then, under some growth assumption on the norm of these controls, a limit motion exists, which satisfies the sliding condition.

\section{Discontinuous distributed control for linear systems}

In what follows we will be concerned with linear controlled differential equations of the form

$$
\left\{\begin{array}{l}
\dot{x}(t)=A x(t)+B u(x(t)) \\
x(0)=x_{0}
\end{array}\right.
$$

where $x$ is the state variable and $u$ is the control variable. The following conditions are assumed to hold:

Hypothesis 2.1.

$011 / 2$ 
(i) $A: \mathscr{D}(A) \subset X \rightarrow X$ is a linear (possibly unbounded) operator, which is the infinitesimal generator of a $C_{0}$-semigroup $K(t), t \geq 0$, on the reflexive Banach space $X$.

(ii) $U$ is a Banach space and $B: U \rightarrow X$ is a continuous linear operator.

(iii) $u: \mathscr{D}(u) \subset X \rightarrow U$ is a densely defined function that satisfies the growth condition

$$
\|u(x)\| \leq M\|x\|+N, \quad \forall x \in \mathscr{D}(u)
$$

for some positive constants $M$ and $N$.

\subsection{Generalized solutions and viability}

We now summarize some results regarding the definition of generalized solution introduced in [6] for discontinuous autonomous semilinear differential equations in Banach spaces.

In our framework, let us define the set-valued map $F: X \rightarrow 2^{X}$ by

$$
F(x)=\bigcap_{\varepsilon>0} \overline{\operatorname{co}} B u(\overline{\mathscr{B}}(x, \varepsilon) \cap \mathscr{D}(u)), \quad x \in X,
$$

where $\overline{\mathscr{B}}(x, r)$ is the closed ball of center $x$ and radius $r$. It is not difficult to prove that this is a well-defined closed and convex valued mapping which is strongly-weakly upper semicontinuous, i.e. for all $x \in X$ and any neighbourhood $V$ of $F(x)$ in the weak topology of $X$, there exists a strong neighbourhood $W$ of $x$ such that $F(y) \subset V$ for any $y \in W$. We use now this set-valued function to generalize the concept of solution.

We will call generalized solution of the differential equation

$$
\left\{\begin{array}{l}
\dot{x}(t)=A x(t)+u(x(t)) \\
x(0)=x_{0}
\end{array}\right.
$$

a mild solution of the differential inclusion

$$
\left\{\begin{array}{l}
\dot{x}(t)-A x(t) \in F(x(t)) \\
x(0)=x_{0} .
\end{array}\right.
$$

A continuous function $x:[0, T] \rightarrow X$ is called a mild solution of (2.5) if there exists $g \in L^{1}(0, T ; X)$ with $g(s) \in F(x(s))$ for almost all $s \in[0, T]$ such that

$$
x(t)=K(t) x_{0}+\int_{0}^{t} K(t-s) g(s) d s, \quad t \in[0, T]
$$

(see i.e. [18, 2] and references therein for a discussion about mild solutions and existence theorems).

In what follows, we will be particularly interested in the following class of solutions: if $S$ is a subset of $X$ and $x_{0} \in S$ a mild solution of inclusion (2.5) that satisfies $x(t) \in S$ for all $t>0$ is called viable on $S . S$ is a viable domain for (2.5) if for any $x_{0} \in S$ there exists a viable solution of the differential inclusion starting from $x_{0}$. A generalized viable solution of (2.4) is a viable solution of (2.5).

The results by Cârjă and Vrabie in [2, 3] can be applied to our differential inclusion, so that we have necessary and sufficient conditions for the existence of viable generalized solutions. 


\subsection{Sliding modes: equivalent control and generalized solutions}

From now on we restrict our attention to a particular class of functions $u$.

Hypothesis 2.2. Let $Y$ be a Banach space, $C: X \rightarrow Y$ a continuous linear operator, $C \neq 0$ and $\mathscr{D}(u)=X \backslash S, S=\operatorname{ker} C$.

This way $S$ is a proper linear subspace of $X$, therefore has void interior so that $\mathscr{D}(u)$ is dense. A sliding mode is attained when, upon reaching the surface $S$, the state is henceforth constrained to remain (slide) on it. Note that the surface $S$ is not given by the problem, but is one of the control tools. The operator $C$ has to be chosen in such a way that, once the evolution is constrained on the sliding surface, the control goal is fulfilled.

In the finite dimensional setting a key concept is that of equivalent control. When it exists, it is defined as the control for which the equation $C \dot{x}=0$ is satisfied. Because of the unboundedness of operator $A$, we have to impose the following conditions to be able to give an extended definition for the infinite dimensional setting.

Hypothesis 2.3. $S \cap \mathscr{D}(A)$ is dense in $S$ in the topology inherited by $X$;

Hypothesis 2.4. the operator $C B: U \rightarrow C B(U) \subset Y$ is continuously invertible and $X=S \oplus B(U)$. The operators $Q=B(C B)^{-1} C$ and $P=I-Q$ will thus be the projections on $B(U)$ along $S$ and vice versa respectively.

We now define the equivalent control $u_{\mathrm{eq}}$ as

$$
u_{\mathrm{eq}}(x):=(C B)^{-1} C A x, \quad \forall x \in S \cap \mathscr{D}(A) .
$$

The projected equation we get substituting $u_{\mathrm{eq}}$ in (2.1) is

$$
\left\{\begin{array}{l}
\dot{x}=(A-Q A) x \\
x(0)=x_{0} \in S .
\end{array}\right.
$$

Proposition 2.1. Let Hypotheses 2.1, 2.2 and 2.3 2.4 hold. Moreover assume that QA is a perturbation of Miyadera-Voigt type satisfying the following condition: there exist $t_{0}>0$ and $q \in[0,1)$ such that

$$
\int_{0}^{t_{0}}\|Q A K(s) x\| d s \leq q\|x\|, \quad \text { for all } x \in \mathscr{D}(A) .
$$

Then the operator $(A-Q A)$ is the generator of a $C_{0}$-semigroup on $X$ and its restriction on $S$ $\tilde{A}: S \cap \mathscr{D}(A) \rightarrow S$ is the generator of a $C_{0}$-semigroup $\tilde{K}(t), t \geq 0$ on $S$.

Proof. Condition (2.8) assures that $A-Q A$ generates a $C_{0}$-semigroup $H(t), t \geq 0$ on $X$ by the perturbation theorem of Miyadera and Voigt (for a proof see for example [4], Section III.3.c). It is easy to prove that $S$ is $H(t)$-invariant, so that the restriction $\tilde{K}(t)$ of $H(t)$ on $S$ is a semigroup on $S$ generated by $\tilde{A}$. The invariance of $H(\cdot)$ is equivalent to this property: there exists $\omega \in \mathbb{R}$ such that for any $\lambda>\omega$ one has $\mathscr{R}(\lambda ; A-Q A) S \subset S$ ([16] Theorem 5.1 p. 121). If $y=\mathscr{R}(\lambda ; A-Q A) x$ for some $x \in S$, then $\lambda y-A y+Q A y=x$. Applying $C$ we get $\lambda C y=0$, therefore $y \in S$ if $\lambda \neq 0$ and the proof is complete. 
We will now show that, under the same set of hypotheses due for the definition of the extended equivalent control, we can say that if there exists a generalized solution viable on $S$ of (2.1), then it is unique and it is consistent with our extended equivalent control method.

Lemma 2.1 (Corollary 3.16 p. 199 in [屯]) Let $A$ generate the $C_{0}$-semigroup $K(t), t \geq 0$ on $X$ and $Q$ be a continuous linear operator such that $Q A$ satisfies condition (2.8) for some $t_{0}>0$ and $q \in$ $[0,1)$. Then the semigroup $\tilde{K}(t), t \geq 0$ generated by $A-Q A$ satisfies

$$
\begin{gathered}
\tilde{K}(t) x=K(t) x+\int_{0}^{t} K(t-s) Q A \tilde{K}(s) x d s \\
\int_{0}^{t_{0}}\|Q A \tilde{K}(s) x\| d s \leq \frac{q}{1-q}\|x\|,
\end{gathered}
$$

for all $x \in \mathscr{D}(A)$, and any $t \geq 0$.

Theorem 2.1. Suppose $X$ is a reflexive Banach space and $f:[0, T] \rightarrow X$ is in $L^{1}(0, T ; X)$. Then there exists a unique function $x:[0, T] \rightarrow X$ which is weakly continuous and such that for each $y \in \mathscr{D}\left(A^{*}\right)$ one has

$$
\langle x(t), y\rangle=\left\langle x_{0}, y\right\rangle+\int_{0}^{t}\left\langle x(s), A^{*} y\right\rangle d s+\int_{0}^{t}\langle f(s), y\rangle d s, \quad 0 \leq t \leq T
$$

and this function is given by

$$
x(t)=K(t) x_{0}+\int_{0}^{t} K(t-s) f(s) d s .
$$

PROOF. See Theorem 4.8.3 and Corollary 4.8.1 in [1], p. 204-205.

Theorem 2.2. Let the hypotheses in Proposition 2.1 be verified. Let $x \in S$ and $z(t)=\tilde{K}(t) x$ for $t \geq 0$ be the mild solution of $\dot{z}=\tilde{A} z, z(0)=x$. Then there exist $f \in L^{1}(0, T ; X)$ such that $z$ is a mild solution of $\dot{z}=A z+f, z(0)=x$.

Proof. By Hypothesis 2.3 there exist a sequence $\left\{x_{n}\right\}$ in $\mathscr{D}(A) \cap S$ such that $x_{n} \rightarrow x$. Setting $z_{n}(t)=\tilde{K}(t) x_{n}$, by (2.9) and (2.11) one has

$$
\left\langle z_{n}(t), y\right\rangle=\left\langle x_{n}, y\right\rangle+\int_{0}^{t}\left\langle z_{n}(s), A^{*} y\right\rangle d s-\int_{0}^{t}\left\langle Q A z_{n}(s), y\right\rangle d s,
$$

for all $t \geq 0$ and $y \in \mathscr{D}\left(A^{*}\right)$. As $\tilde{K}(t), t \geq 0$ is a $C_{0}$-semigroup it follows that $\left\|z_{n}(t)-z(t)\right\| \rightarrow 0$ uniformly on compact subsets of $[0,+\infty)$, therefore

$$
\lim _{n \rightarrow+\infty} \int_{0}^{t}\left\langle Q A z_{n}(s), y\right\rangle d s=-\langle z(t), y\rangle+\langle x, y\rangle+\int_{0}^{t}\left\langle z(s), A^{*} y\right\rangle d s .
$$

For any $t \geq 0$ and any $x$ the vector $\int_{0}^{t} \tilde{K}(s) x d s$ is in $\mathscr{D}(\tilde{A}) \subset \mathscr{D}(A)$ and $\tilde{K}(t) x-x=\tilde{A} \int_{0}^{t} \tilde{K}(s) x d s$, thus

$$
\int_{0}^{t}\left\langle z(s), A^{*} y\right\rangle d s=\left\langle(\tilde{A}+Q A) \int_{0}^{t} z(s) d s, y\right\rangle=\langle z(t), y\rangle-\langle x, y\rangle+\left\langle Q A \int_{0}^{t} z(s) d s, y\right\rangle .
$$


Combining the above results, by the density of $\mathscr{D}\left(A^{*}\right)$ it follows that

$$
\int_{0}^{t} Q A z_{n}(s) d s \rightarrow Q A \int_{0}^{t} z(s) d s, \quad \text { for all } t \geq 0 .
$$

Note that this just depends on the fact that $\tilde{A}$ is a generator and a perturbation of $A$. Condition (2.10) will now be exploited to show that the above convergence holds also in the abstract Sobolev space $W^{1,1}(0, T ; X)$, thus proving the thesis. To simplify notations let $f_{n}(t)=\int_{0}^{t} Q A z_{n}(s) d s$ and $f(t)=Q A \int_{0}^{t} z(s) d s$ for $t \geq 0$. Obviously $f_{n} \in A C(0, T ; X)$ for any $n$ and $T>0$ by the absolute continuity of the Bochner integral. Moreover by (2.10), for $T \leq t_{0}$ and any $n, m$

$$
\left\|f_{n}^{\prime}-f_{m}^{\prime}\right\|_{L^{1}(0, T ; X)}=\int_{0}^{T}\left\|Q A \tilde{K}(s)\left(x_{n}-x_{m}\right)\right\| d s \leq \frac{q}{1-q}\left\|x_{n}-x_{m}\right\| .
$$

Therefore $\left\{f_{n}^{\prime}\right\}$ is a Cauchy sequence in $L^{1}(0, T ; X)$ and since this space is complete, there exists $h \in L^{1}(0, T ; X)$ such that $f_{n}^{\prime} \rightarrow h$ in $L^{1}(0, T ; X)$. Using the same arguments it is easy to see that $\left\{f_{n}\right\}$ is convergent in $L^{1}(0, T ; X)$ and by (2.13) the limit has to be $f$. The only thing to prove now is that in fact $f$ is absolutely continuous and $h=f^{\prime}$ almost everywhere. This can be done by a standard argument involving derivatives in the distribution sense, applied to the abstract setting. In fact let $\mathscr{D}^{\prime}(0, T ; X)$ be the space of $X$-valued distributions on $(0, T)$, i.e. $\mathscr{D}^{\prime}(0, T ; X)=\mathscr{L}(\mathscr{D}(0, T), X)$. The derivative of a distribution in $\mathscr{D}^{\prime}(0, T ; X)$ is defined in the usual way and for $f \in \in^{1}(0, T ; X)$, $\varphi \in \mathscr{D}(0, T)$ it gives

$$
\left(\frac{d}{d t} f\right)(\varphi):=-f\left(\varphi^{\prime}\right):=-\int_{0}^{T} f(s) \varphi^{\prime}(s) d s
$$

Therefore for any $\varphi \in \mathscr{D}(0, T)$

$$
-\int_{0}^{T} f(s) \varphi^{\prime}(s) d s=\lim -\int_{0}^{T} f_{n}(s) \varphi^{\prime}(s) d s=\lim \int_{0}^{T} f_{n}^{\prime}(s) \varphi(s) d s=\int_{0}^{T} h(s) \varphi(s) d s,
$$

that is $f^{\prime}=h$ in $L^{1}(0, T ; X)$.

From (2.12) it then follows that

$$
\langle z(t), y\rangle=\langle x, y\rangle+\int_{0}^{t}\left\langle z(s), A^{*} y\right\rangle d s-\int_{0}^{t}\langle h(s), y\rangle d s,
$$

where $h \in L^{1}(0, T ; X)$

$$
h(s)=\frac{d}{d s} Q A \int_{0}^{s} z(r) d r, \quad \text { a. e. } s \in[0, T]
$$

and the thesis follows from Theorem 2.1.

Remark 2.1. Similar results have been previously proved, under stronger hypotheses. In [6] the operator $A$ was assumed to generate a compact semigroup, while in [5] the requirement was the extendability on $S$ of the operator $Q A$. Suppose that $U$ is finite-dimensional, or for simplicity that the control is scalar. Then $C x=\langle\gamma, x\rangle$ for some $\gamma \in X^{*}$ and $Q A$ admits an extension iff $\gamma \in \mathscr{D}\left(A^{*}\right)$, while this condition is not required for (2.8) to be verified. 


\section{Discontinuous Neumann boundary control}

In Section 1 we considered linear systems of the form (2.1) and we assumed in Hypothesis 2.1-(ii) that the control is distributed. Now we consider a class of parabolic partial differential equations (possibly non-linear) with controllers acting on the boundary. In particular we will study the case of Neumann boundary conditions and finite dimensional control space. Also, we suppose that a manifold $S$ is given, on which we want to restrict the motion of our system. We analyse the problem of the existence of an admissible control law for which this ideal sliding motion is possible.

\subsection{Variational formulation}

The setting of the abstract problem follows [7, 8, 9]: let $V$ be a separable, reflexive Banach space, $H$ be a Hilbert space, $V \subset H$ with continuous injection. The space $H$ is identified with its dual, while we denote by $V^{\prime}$ the dual space of $V$, so that we have

$$
V \subset H \subset V^{\prime}
$$

For $u_{1}, u_{2} \in H$ the scalar product in $H$ will be denoted by $\left(u_{1}, u_{2}\right)$ and the derived norm by $\left|u_{i}\right|$. We will denote by $\|\cdot\|$ the norm in $V$ and by $\|\cdot\|_{*}$ that in $V^{\prime}$. The dual pairing between the two spaces will be written as $\langle\cdot, \cdot\rangle$. Also, we will assume that on $V$ it is defined a semi-norm [.] such that

$$
[v]+\lambda|v| \geq \beta\|v\|, \quad \forall v \in V, \quad \text { for some } \lambda, \beta>0 .
$$

It is assumed that all the above (infinite-dimensional) spaces are real vector spaces; results can be extended to the complex case with the necessary modifications. For any $T>0$ we can define the following spaces of vector-valued functions:

$$
\begin{aligned}
& L^{2}(0, T ; V)=\left\{f:[0, T] \rightarrow V:\|f\|_{2, V}^{2}:=\int_{0}^{T}\|f(t)\|^{2} d t<+\infty\right\} \\
& L^{\infty}(0, T ; H)=\left\{f:[0, T] \rightarrow H:\|f\|_{\infty, H}^{2}:=\sup _{t \in[0, T]}|f(t)|<+\infty\right\} .
\end{aligned}
$$

The space $L^{2}\left(0, T ; V^{\prime}\right)$ can be defined analogously. Also, it is possible to define on these spaces a concept of derivative, in a distributional sense (see i. e. [8] Chapter III). The following result [9] will be useful in the sequel.

Theorem 3.1. Let

$$
W(0, T)=\left\{f \in L^{2}(0, T ; V): \frac{d f}{d t} \in L^{2}\left(0, T ; V^{\prime}\right)\right\} .
$$

All functions in $W(0, T)$ are, after eventual modification on a null measure set, continuous from $[0, T]$ in $H$, i.e. $W(0, T) \subset C^{0}(0, T ; H)$.

Hypothesis 3.1. For $t \in(0, T)$ let $A(t): V \rightarrow V^{\prime}$ be an operator satisfying the following assumptions:

- for all $v, w \in V$ the map

$$
t \mapsto\langle A(t) v, w\rangle \text { is measurable; }
$$


- for all $t$ and any $u, v, \omega \in V$ the map

$$
\alpha \mapsto\langle A(t)(u+\alpha v), w\rangle \text { is continuous; }
$$

- there exist constants $c_{1}>0, c_{2} \geq 0$ such that

$$
\|A(t) v\|_{*} \leq c_{1}\|v\|+c_{2}, \quad \forall v \in V
$$

- there exist constants $\alpha>0$ and $v \in \mathbb{R}$ such that

$$
\langle A(t) v, v\rangle \geq \alpha[v]^{2}+v|v|^{2} \forall v \in V
$$

- $A(\cdot)$ is 2-weakly continuous, i.e.

$$
v_{k} \rightarrow v \text { weakly in } W(0, T) \Longrightarrow A(\cdot) v_{k}(\cdot) \rightarrow A(\cdot) v(\cdot) \text { weakly in } L^{2}\left(0, T ; V^{\prime}\right) .
$$

Let $U \subset \mathbb{R}^{m}$ be closed and convex and let $f:[0, T] \times U \rightarrow V^{\prime}$ be measurable in $t \in[0, T]$ for all $u \in U$ and linear in $u$ for all $t$. Since it will always be understood that admissible control laws $u$ take values in $U$, we will write $u \in L^{2}(0, T)$ instead of $L^{2}(0, T ; U)$ and denote by $\|u\|_{2}$ the usual $L_{2}$-norm of the function $u$.

We are now ready to write the abstract evolution equation we are going to study. The evolution of the system will be given by a vector-valued function $y \in W(0, T)$ satisfying the following abstract Cauchy problem

$$
\left\{\begin{array}{l}
\frac{d y}{d t}+A(t) y(t)=f(t, u(t)) \quad \text { a.e. } t \\
y(0)=y_{0}
\end{array}\right.
$$

with $u \in L^{2}(0, T)$ and for some $y_{0} \in H$ (by Theorem 3.1 this makes sense). The differential equation above as to be understood as an equality in the dual space $V^{\prime}$, i.e. setting

$$
a(t ; v, w)=\langle A(t) v, w\rangle, \quad t>0, \quad v, w, \in V
$$

and in view of Theorem 3.1, the differential problem (3.7) is equivalent to the following variational formulation

$$
\left\{\begin{array}{l}
\frac{d}{d t}(y(t), v)+a(t ; y(t), v)=\langle f(t, u(t)), v\rangle \quad \forall v \in V, \\
y(0)=y_{0}
\end{array}\right.
$$

Existence and uniqueness results of the solution of such equations, under our assumptions, can be found in [7] under monotonicity assumptions and in [8, 6] for the linear case.

Assume we are working in the framework set up in this section. Thanks to separability, there exists a countable basis for $V$, so that it is possible to define a family $\left\{V_{k}\right\}_{k \in \mathbb{N}}$ of finite dimensional subspaces of $V$

$$
V_{k}=\operatorname{span}\left\{v_{1, k}, \ldots, v_{N_{k}, k}\right\}
$$

such that

$$
V_{k} \subset V_{k+1}, \quad \bigcup_{k \in N} V_{k}=V
$$


Then it is possible to define approximate solutions of (3.9) by projecting on the subspaces $V_{k}$, using the standard Faedo-Galerkin method. We thus define the following family of variational problems: find $y_{k}:[0, T] \rightarrow V_{k}$ such that

$$
\left\{\begin{array}{l}
\frac{d}{d t}\left(y_{k}(t), v\right)+a\left(t ; y_{k}(t), v\right)=\left\langle f\left(t, u_{k}(t)\right), v\right\rangle \quad \forall v \in V_{k}, \\
y_{k}(0)=y_{0, k}
\end{array}\right.
$$

with $y_{0, k} \in V_{k}$ for all $k$ and a sequence $\left\{u_{k}\right\}$ in $L^{2}(0, T)$.

We now prove a convergence result for the approximations $y_{k}$, under some conditions on the controls sequence $\left\{u_{k}\right\}$.

Theorem 3.2. Let the assumptions in Section 3 be satisfied and $\left\{u_{k}\right\}$ be a sequence in $L^{2}(0, T)$. Let $y_{k}$ be the solution of (3.10) and suppose that $y_{0, k} \rightarrow y_{0}$ in $H$ for $k \rightarrow+\infty$. Suppose moreover that the following condition on the growth of the control norms is satisfied

$$
\left\|u_{k}\right\|_{L^{2}(0, t)}^{2} \leq M \int_{0}^{t}\left|y_{k}(s)\right|^{2} d s+N, \quad t \leq T
$$

for some non-negative constants $M$ and $N$ and that there exist a constant $C>0$ such that for any $u \in L^{2}(0, T)$

$$
\int_{0}^{T}\|f(t, u(t))\|_{*}^{2} d t \leq C\|u\|_{2}^{2} .
$$

Then there exist a control law $u^{*} \in L^{2}(0, T)$ and a function $y^{*} \in W(0, T)$ verifying (3.9), such that, for some subsequence,

$$
\begin{aligned}
& y_{k} \rightarrow y^{*} \text { weakly in } W(0, T) \\
& y_{k} \rightarrow y^{*} \text { weakly* in } L^{\infty}(0, T ; H) \\
& u_{k} \rightarrow u^{*} \text { weakly in } L^{2}(0, T) .
\end{aligned}
$$

PROOF. Writing (3.10) for $v=y_{k}(t)$ we get

$$
\left(\dot{y}_{k}(t), y_{k}(t)\right)+a\left(t ; y_{k}(t), y_{k}(t)\right)=\left\langle f\left(t, u_{k}(t)\right), y_{k}(t)\right\rangle \text {. }
$$

As the first term on the left is in fact the time derivative of $\left|y_{k}(t)\right|^{2} / 2$, integrating the above identity we have

$$
\frac{1}{2}\left|y_{k}(t)\right|^{2}+\int_{0}^{t} a\left(t ; y_{k}(s), y_{k}(s)\right) d s=\frac{1}{2}\left|y_{k}(0)\right|^{2}+\int_{0}^{t}\left\langle f\left(t, u_{k}(s)\right), y_{k}(s)\right\rangle d s .
$$

By (3.5), (3.1) and (3.12) we obtain the following inequality

$$
\begin{aligned}
\frac{1}{2}\left|y_{k}(t)\right|^{2}+\alpha \int_{0}^{t}\left[y_{k}(s)\right]^{2} d s \leq \frac{1}{2}\left|y_{k}(0)\right|^{2}-v \int_{0}^{t}\left|y_{k}(s)\right|^{2} d s & \\
& +c\left\|u_{k}\right\|_{2}\left(\int_{0}^{t}\left|y_{k}(s)\right|^{2} d s+\int_{0}^{t}\left[y_{k}(s)\right]^{2} d s\right)^{1 / 2}
\end{aligned}
$$


for some constant $c>0$. Consider now for $x \geq 0$ the function $h(x)=(\alpha x) / 2-c \sqrt{x}$. It is easy to show that it has minimum for $x=(c / \alpha)^{2}$, therefore $c \sqrt{x} \leq\left(\alpha x+c^{2} / \alpha\right) / 2$, thus

$$
\frac{1}{2}\left|y_{k}(t)\right|^{2}+\frac{\alpha}{2} \int_{0}^{t}\left[y_{k}(s)\right]^{2} d s \leq \frac{1}{2}\left|y_{k}(0)\right|^{2}+\left(\frac{\alpha}{2}+|v|\right) \int_{0}^{t}\left|y_{k}(s)\right|^{2} d s+\frac{c^{2}}{2 \alpha}\left\|u_{k}\right\|_{2}^{2} .
$$

Now, since by hypothesis $\left|y_{0, k}-y_{0}\right|$ tends to zero, the term $\left|y_{k}(0)\right|^{2}$ is bounded. Moreover by (3.11)

$$
\left|y_{k}(t)\right|^{2}+\alpha \int_{0}^{t}\left[y_{k}(s)\right]^{2} d s \leq c_{1}+c_{2} \int_{0}^{t}\left|y_{k}(s)\right|^{2} d s
$$

for some constants $c_{1}, c_{2}>0$. Since $\alpha>0$ we get

$$
\left|y_{k}(t)\right|^{2} \leq c_{1}+c_{2} \int_{0}^{t}\left|y_{k}(s)\right|^{2} d s
$$

Therefore, by Gronwall's lemma we obtain for some constant $K>0$

$$
\left\|y_{k}\right\|_{L^{\infty}(0, T ; H)}=\sup _{t \in[0, T]}\left|y_{k}(t)\right| \leq K
$$

therefore from (3.13) we also have

$$
\int_{0}^{T}\left[y_{k}(s)\right]^{2} d s \leq \mathrm{const}
$$

and lastly, using (3.1) and (3.4)

$$
\begin{aligned}
\left\|y_{k}\right\|_{L^{2}(0, T ; V)}= & \left(\int_{0}^{T}\left\|y_{k}(s)\right\|^{2} d s\right) \leq \mathrm{const}, \\
& \int_{0}^{T}\left\|A(t) y_{k}(t)\right\|_{*} d t \leq \mathrm{const} .
\end{aligned}
$$

Since spheres are weakly compact in both $L^{2}(0, T ; V)$ and $L^{2}\left(0, T ; V^{\prime}\right)$ and weakly* compact in $L^{\infty}(0, T ; H)$, we can extract a subsequence of $\left\{y_{k}\right\}$ (which for simplicity we still denote by $\left\{y_{k}\right\}$ ) converging to some $y^{*} \in L^{2}(0, T ; V) \cap L^{\infty}(0, T ; H)$ for both the weak topology of $L^{2}(0, T ; V)$ and the weak* topology of $L^{\infty}(0, T ; H)$ and such that $A y_{k}$ weakly converges to some $\eta$ in $L^{2}\left(0, T ; V^{\prime}\right)$. By (3.11) we also have that $\left\|u_{k}\right\|_{2}$ is bounded, thus eventually passing to a further subsequence, there exists $u^{*} \in L^{2}(0, T)$ such that $u_{k}$ converges to $u^{*}$ weakly in $L^{2}(0, T)$. Also, by linearity of $f$ we can proceed as in the proof of Theorem 1.1, p. 159 of [7] to conclude that

$$
\left\{\begin{array}{l}
\frac{d}{d t} y^{*}(t)+\eta(t)=f\left(t, u^{*}(t)\right) \\
y(0)=y_{0}
\end{array}\right.
$$

Also, by a standard argument (see i.e. [19], Theorem 3) one can prove that $\dot{y}_{k} \rightarrow \dot{y}^{*}$ weakly in $L^{2}\left(0, T ; V^{\prime}\right)$, i.e. $y_{k} \rightarrow y^{*}$ weakly in $W(0, T)$. Thus, by (3.6) $\eta(t)=A(t) y^{*}(t)$ and the proof is complete.

Having achieved the above convergence result, we introduce as in [19], a set $D$ which can be either $V$ or a sufficiently large open subset of $H$ and a mapping $s: D \rightarrow \mathbb{R}^{m}$ continuously Fréchet differentiable on $D$. The sliding surface $S$ we consider is defined as $S=\{y \in D: s(y)=0\}$. Proceeding as in [19], by slightly modifying proofs, it is possible to show the following 
Corollary 3.1. Let the assumptions of Theorem 3.2 hold. Let $z_{k}(t)=s\left(y_{k}(t)\right)$ and assume that one of the following is satisfied:

(1) $D=V$, $s$ is affine and $z_{k} \rightarrow 0$ uniformly in $t$;

(2) $\mathscr{B}_{H}(0, K) \subset D, V$ is compactly embedded in $H$ (here $\mathscr{B}_{H}$ denotes a ball in $H$, while $K$ is defined in (3.14) above) and $z_{k}(t) \rightarrow 0$ for almost every $t \in[0, T]$.

Then the limit motion $y^{*}$ of Theorem 3.2 belongs to the sliding manifold S.

Remark 3.1. Note that every $y_{k}$ solves a finite-dimensional problem, thus for the approximate solutions all results of the classical theory of variable structure systems and sliding mode control of [17] are valid. Therefore existence results for system motions satisfying the requirements in Corollary 3.1 and design methods to achieve them are available. See also the discussion of existence under relaxed hypotheses developed in [19].

\section{Conclusions and future work}

In this paper we showed how the use of discontinuous controls can be validated in an infinite dimensional setting. For the linear, distributed case a semigroup approach combined with a definition of generalised solution allows the interpretation of sliding modes as viable generalized solutions of discontinuous differential equations.

As for linear boundary controls, we have analysed the convergence behaviour of finite dimensional Faedo-Galerkin approximations for a class of variational problems, when sliding motions are taken into consideration. Under some growth hypothesis on the norms of the controls, a sliding motion exists.

While the notion of equivalent control has been extended quite simply in the first case, it would be interesting to study how the results for boundary controls are related to a similar concept and to study approximability of ideal sliding motions by real ones in this context.

\section{References}

[1] A. V. Balakrishnan, Applied functional analysis, Springer-Verlag, 1976, Applications of Mathematics, No. 3.

[2] Ovidiu Cârjă and Ioan I. Vrabie, Some new viability results for semilinear differential inclusions, NoDEA Nonlinear Differential Equations Appl. 4 (1997), no. 3, 401-424.

[3] __ Viability for semilinear differential inclusions via the weak sequential tangency condition, J. Math. Anal. Appl. 262 (2001), no. 1, 24-38.

[4] Klaus-Jochen Engel and Rainer Nagel, One-parameter semigroups for linear evolution equations, Graduate Texts in Mathematics, vol. 194, Springer-Verlag, 2000.

[5] Laura Levaggi, Infinite dimensional systems'sliding motions., Eur. J. Control 8 (2002), no. 6, $508-516$.

[6] ___ Sliding modes in banach spaces., Differ. Integral Equ. 15 (2002), no. 2, 167-189.

[7] J.-L. Lions, Quelques méthodes de résolution des problèmes aux limites non linéaires, Dunod, Paris, 1969. 
[8] J. L. Lions, Optimal control of systems governed by partial differential equations, Die Grundlehren der mathematischen Wissenschaften, Band 170, Springer-Verlag, New York, 1971.

[9] J.-L. Lions and E. Magenes, Non-homogeneous boundary value problems and applications. vol. $i$, Die Grundlehren der mathematischen Wissenschaften, Band 181, Springer-Verlag, New York, 1972.

[10] Yu. V. Orlov and V. I. Utkin, Use of sliding modes in distributed system control problems, Automat. Remote Control 43 (1982), no. 9, 1127-1135 (1983).

[11] _ Sliding mode control in indefinite-dimensional systems, Automatica J. IFAC 23 (1987), no. 6, $753-757$.

[12] Yuri Orlov and Denis Dochain, Discontinuous feedback stabilization of minimum-phase semilinear infinite-dimensional systems with application to chemical tubular reactor, IEEE Trans. Automat. Control 47 (2002), no. 8, 1293-1304.

[13] Yuri Orlov and Vadim I. Utkin, Unit sliding mode control in infinite-dimensional systems, Appl. Math. Comput. Sci. 8 (1998), no. 1, 7-20.

[14] Yuri V. Orlov, Discontinuous unit feedback control of uncertain infinite-dimensional systems, IEEE Trans. Automat. Control 45 (2000), no. 5, 834-843.

[15] Yury Orlov, Yiming Lou, and Panagiotis D. Christofides, Robust stabilization of infinite-dimensional systems using sliding-mode output feedback control, Internat. J. Control 77 (2004), no. 12, 1115-1136.

[16] A. Pazy, Semigroups of linear operators and applications to partial differential equations, Applied Mathematical Sciences, vol. 44, Springer-Verlag, New York, 1983.

[17] Vadim I. Utkin, Sliding modes in control and optimization, Communications and Control Engineering Series, Springer-Verlag, Berlin, 1992.

[18] I. I. Vrabie, Compactness methods for nonlinear evolutions, Pitman Monographs and Surveys in Pure and Applied Mathematics, vol. 32, Longman Scientific \& Technical, Harlow, 1987.

[19] Tullio Zolezzi, Variable structure control of semilinear evolution equations, in Partial differential equations and the calculus of variations - Vol. II, Progr. Nonlinear Differential Equations Appl., Birkhäuser, Boston, MA, 1989, pp. 997-1018. 